\title{
17 SALUd MENTAL POSITIVA: DEL CONCEPTO AL CONSTRUCTO. EVOLUCIÓN HISTÓRICA Y REVISIÓN DE TEORÍAS
}

\author{
| Jaione Santiago'; Elena Bernaras²; Joana Jaureguizar ${ }^{3}$ |
}

\section{RESUMEN}

CONTEXTO: Definir la Salud Mental Positiva (SMP) y conocer la "etiología de la Salud Mental" sigue resultando difícil. Los estudios para conceptualizar y operacionalizar la promoción de la Salud Mental han aumentado exponencialmente, originándose múltiples conceptos afines como Bienestar Psicológico, Florecimiento o SMP.

OBJETIVO(S): (1) Analizar la SMP como concepto para describir su evolución histórica. (2) Analizar la SMP como constructo, explorando en paralelo sus dimensiones y factores propuestos en los diversos modelos teóricos existentes.

METODOLOGÍA: Revisión de la literatura mediante búsqueda en las bases de datos Web of Science, PubMed, Scielo, PsycINFO y Psicodoc, cuyos criterios de inclusión fue: estar escritos en inglés, español o portugués, disponibles en full-text y cuya temática trate de la SMP. Los términos de búsqueda fueron: Salud Mental Positiva, Promoción de la Salud Mental, Bienestar, Bienestar Psicológico, Bienestar Emocional y Flourish.

RESULTADOS: Se obtuvieron un total de 128 artículos que daban respuesta a los objetivos, aunque finalmente se utilizaron 20 para la realización de este trabajo. Tras el análisis categorial de la información obtenida, se establecieron dos apartados para dar respuesta a los objetivos. CONCLUSIONES: La SMP como concepto ha encontrado su espacio en el ámbito de la Salud Pública, y de la Psicología, siendo equiparable a términos como Bienestar o Flourish. Como constructo, Hedonía y Eudamonía se constituyen como sus principales componentes.

\section{DESCRIPTORES: Salud mental; Promoción de la salud; Teoría psicológica; Revisión}

\section{RESUMO}

"Saúde mental positiva: Do conceito ao construto. Evolução histórica e revisão de teorías"

CONTEXTO: Definir a Saúde Mental Positiva (SMP) e conhecer a "etiologia da Saúde Mental" permanece difícil. Estudos para conceptualizar e operacionalizar a promoção da Saúde Mental têm aumentado exponencialmente, dando origem a múltiplos conceitos relacionados, tais como bem-estar psicológico, "flourish" ou SMP.

OBJETIVO(S): (1) Analisar a SMP como um conceito para descrever sua evolução histórica. (2) Analisar a SMP como construto, explorando em paralelo dimensões e fatores propostos nos diversos modelos teóricos existentes.

MÉTODOS: Revisão da literatura por meio de busca nas bases de dados Web of Science, PubMed, Scielo, PsycINFO e Psicodoc, cujos critérios de inclusão foram: ser escrito em inglês, espanhol ou português, disponível em texto integral e cujo assunto trata da SMP. Os termos da pesquisa foram: Saúde Mental Positiva, Promoção da Saúde Mental, Bem-estar, Bem-estar Psicológico, Bem-estar Emocional e Flourish.

RESULTADOS: Foram obtidos um total de 128 artigos que responderam aos objetivos, embora 20 tenham sido utilizados para a realização deste trabalho. Após a análise categorial das informações obtidas, foram estabelecidas duas secções para responder aos objetivos.

CONCLUSÕES: A SMP como conceito tem encontrado seu lugar no campo da Saúde Pública e Psicologia, sendo comparável a termos como Bem-estar ou Flourish. Como construto, Hedonía e Eudamonía são seus principais componentes.

PALAVRAS-CHAVE: Saúde mental; Promoção da saúde; Teoria psicológica; Revisão

\section{ABSTRACT}

"Positive mental health: From the concept to the construct. Historic evolution and theories review"

BACKGROUND: Defining Positive Mental Health (PMH) and knowing the "etiology of Mental Health" remains difficult. Studies to conceptualize and operationalize the positive aspect of Mental Health have increased exponentially, giving rise to multiple related concepts such as Psychological Wellbeing, Flourishing or PMH.

AIM: (1) To analyze the PMH as a concept, to describe its historical evolution. (2) To analyze the PMH as a construct, exploring in parallel its dimensions and factors proposed in the various existing theoretical models. METHODS: Literature review by means of search in the Web of Science, PubMed, Scielo, PsycINFO and Psicodoc databases, whose inclusion criteria were: to be written in English, Spanish or Portuguese, available in full-text and whose subject matter deals with the SMP. The search terms were: Positive Mental Health, Mental Health Promotion, Well-being, Psychological Well-being, Emotional Well-being and Flourish.

RESULTS: A total of 128 articles were obtained that gave answer to the objectives, although 20 were finally used for the accomplishment of this work. After the categorial analysis of the information obtained, two sections were established to respond to the objectives.

CONCLUSIONS: The PMH as a concept has found its place in the field of Public Health, and Psychology, being comparable to terms such as WellBeing or Flourish. As a construct, Hedonia and Eudamonia constitute themselves as its main components.

\section{KEYWORDS: Mental health; Health promotion; Psychological theory; Review}

Submetido em 30-12-2018

Aceite em 12-03-2019

1 Enfermeira especalista em Enfermagem de Saúde Mental e Psiquiátrica; Professora na Universidad del País Vasco, Escuela Universitaria de Enfermería de Vitoria-Gasteiz, Calle Jose Atxotegi, Vitoria-Gasteiz, Espanha, jaione_santiago001@ehu.eus

2 Doutora em Psicologia; Professora Titular de Universidad na Universidad del País Vasco, Facultad de Educación, Filosofía y Antropología, Espanha, elena.bernaras@ehu.eus 3 Doutora em Psicologia; Profesora Agregada na Universidad del País Vasco, Facultad de Educación, Filosofía y Antropología, Leioa, Espanha, joana.jauregizar@ehu.eus 


\section{INTRODUCCIÓN}

Numerosas disciplinas científicas que estudian la Salud Mental están realizando un cambio hacia un paradigma positivo. Esto podría deberse al aumento progresivo de la prevalencia de las enfermedades mentales (EM) y su gran impacto en la calidad de vida, superior al de enfermedades crónicas como la diabetes o las cardiopatías (OMS, 2017). Por otro lado, numerosos estudios han demostrado que niveles elevados de bienestar están relacionados con resultados positivos como el aumento de productividad, conducta pro-social o aumento de la salud y esperanza de vida, entre otros (Huppert y So, 2013).

Parece necesario, pues, explorar nuevas áreas de actuación más acordes con las líneas estratégicas de salud planteadas por la Organización Mundial de la Salud (OMS, 2013) que suponen una alternativa sólida para incrementar la Salud Mental de las personas y disminuir, en consecuencia, la aparición de algunas EM o las secuelas de las mismas.

\section{Antecedentes y Estado de la Cuestión}

El inicio de esta nueva perspectiva, promovida por la transición epidemiológica-sanitaria, tuvo lugar tras la Segunda Guerra Mundial. Gracias a los avances científico-médicos, se produjo una doble transformación; por un lado, los perfiles de las enfermedades del momento pasaron de ser agudas, infecciosas y letales en su mayoría, a crónicas, originando, en consecuencia, el aumento de la esperanza de vida. Así, se pasó de un Modelo Patogenicista, basado en la concepción de la salud únicamente como la ausencia de enfermedad (Keyes, 2007), a un Modelo Salutogénico propuesto por Antonovsky (1996) y caracterizado por tener como objetivos la atención centrada en la persona y el desarrollo de sus activos de salud (Botello et al., 2013). Por tanto, se le otorgan mayor relevancia a aquellas capacidades humanas que permiten a las personas incrementar el control sobre su salud y se ponen en entredicho los conceptos de salud y enfermedad que hasta el momento prevalecían.

A partir de la segunda mitad del siglo XX, Marie Jahoda utilizó por primera vez el concepto de Salud Mental Positiva (SMP) e identificó 6 componentes clave de la misma (Jahoda, 1958). Posteriormente, referentes como Diener (1984) y su Teoría del Bienestar Subjetivo, Ryff (1989) y su Teoría del Bienestar Psicológico o Seligman (2002), considerado el padre de la Psicología Positiva, se han dedicado a su estudio.
La Enfermería, quien lleva implícita en su ADN profesional la tarea de cuidar y ayudar al individuo a alcanzar el mayor grado de desarrollo personal (Orem y Vardiman, 1994), también ha manifestado interés por el paradigma positivo. Su posicionamiento estratégico en el sistema sanitario la convierte en un agente de salud privilegiado a la hora de interactuar con la comunidad. Así, aboga por la promoción de la salud física o mental no sólo durante la administración de cuidados, sino también a través de actividades dirigidas a reforzar, potenciar y mantener el máximo nivel de salud posible (Wand, 2013).

\section{Objetivos}

Analizar la "Salud Mental Positiva" como concepto, describiendo la evolución histórica del mismo. En segundo lugar, analizar la "Salud Mental Positiva" como constructo, explorando en paralelo las distintas dimensiones propuestas en los principales modelos teóricos.

\section{METODOLOGÍA}

Se ha llevado a cabo una Revisión de la Literatura en las siguientes bases de datos: Web of Science, Google Scholar, Medline, y PsycINFO. Los términos de búsqueda utilizados fueron: Salud Mental Positiva, Promoción de la Salud Mental, Bienestar, Bienestar Psicológico, Bienestar Emocional y Flourish y los criterios de inclusión: tener como tema de investigación la SMP, estar disponible en full-text, y estar escrito en español, inglés o portugués. Asimismo, se estableció un periodo amplio de búsqueda (desde 1950 hasta la actualidad).

\section{RESULTADOS}

Se obtuvieron un total de 128 artículos que cumplían los criterios de inclusión. Para la realización de este trabajo, se seleccionaron 20 artículos priorizando la fecha y tipo de publicación, con el fin de obtener datos actualizados y de la mayor evidencia científica posible. Tras el análisis categorial de la información obtenida, se establecieron dos apartados para dar respuesta a los dos objetivos del estudio. En el primer apartado se explica el desarrollo del concepto a lo largo de las últimas siete décadas, mientras que en el segundo, se ha realizado un estudio comparativo de las principales teorías buscando paralelismos entre las dimensiones que cada una de ellas adjudican a la SMP como constructo. 
Aproximación Conceptual a la Salud Mental Positiva: Evolución Histórica

La conceptualización del término SMP sigue resultando una empresa de difícil concreción. En primer lugar, porque es necesario superar la tendencia patogenicista propia del colectivo sanitario. Como afirman Kobau et al. (2011), este enfoque centrado en las EM -sus causas, síntomas y consecuencias- tiene como resultado la aparición y prolongación en el tiempo de actitudes estigmatizantes entre los y las profesionales de la salud. Asimismo, Lluch-Canut (2008) establece que, si bien la terminología utilizada en nuestro sistema sanitario es positiva, el resto de aspectos vinculados a la salud mental son negativos o inmersos en la enfermedad y el trastorno mental, lo que nos lleva a un uso eufemístico del término.

Por otra parte, encontrar información en Medline ha resultado complejo debido a la ausencia de descriptores en su tesauro. Efectivamente, ningún término relacionado con la SMP, ni tan siquiera el de "bienestar", lo tiene. Esto dificulta la realización de revisiones sistemáticas y refleja que aún prevalece el modelo biomédico-patogenicista.

Por último, el análisis del material obtenido revela numerosos conceptos de significado similar al de la SMP. Así, encontramos términos que se refieren sólo a algún componente de la SMP, como "optimismo", "afectos positivos" o "satisfacción con la vida", mientras que otros, fundamentados en modelos teóricos que los avalan, representan una visión global del concepto. Entre estos últimos encontramos "Salud Mental Positiva", propuesto por Jahoda (1958) y recuperado por Lluch-Canut (2002), "Bienestar", cuyas diversas categorías -bienestar emocional, subjetivo, o psicológicohan sido ampliamente estudiadas por varios autores (Diener, 1984; Ryff, 1989), "Complete Mental Health" sugerido por Keyes (2007) y “Flourish”, en español florecimiento, término rescatado por Seligman (2002) y Diener et al. (2010) para la construcción de sus teorías. De hecho, estos autores y otros referentes en el campo de la Psicología Positiva utilizan indistintamente los términos "Bienestar Global", "Salud Mental Positiva" y "Flourish", considerándolos equivalentes (Diener et al., 2010; Huppert y So, 2013; Keyes y Simoes, 2012; Kobau et al., 2011; OMS, 2013; Seligman, 2012).

\section{Evolución del Concepto “Bienestar”.}

Inicialmente, el análisis del bienestar se centró principalmente en el estudio de la presencia o ausencia de emociones positivas.
Autores como Bradburn (1969) o Diener (1984) realizaron numerosas investigaciones en torno a las emociones positivas y la satisfacción con la vida. Es lo que se conoce con el nombre de bienestar subjetivo, y constituirá uno de los principales elementos del componente hedónico del constructo.

En 1989, Ryff desarrolló nuevas perspectivas del bienestar relacionadas con el funcionamiento óptimo de las personas, dando lugar a la descripción del componente eudamónico del bienestar (Keyes y Simoes, 2012). Sin embargo, éste resultó ser aún insuficiente para otros autores, que destacan la importancia de incluir aspectos sociales que intervienen en el estado de SMP de la persona. Así, Keyes (2007) establece que este segundo componente eudamónico deberá incluir aspectos como el nivel de contribución o integración social, entre otros.

En esta misma línea, destacan cuatro autores que describen en sus modelos teóricos la SMP como un constructo multidimensional: en primer lugar, LluchCanut quien, recuperando el término acuñado por Jahoda en 1958, desarrolla el "Modelo Multifactorial de Salud Mental Positiva” (Lluch-Canut, 2002); Keyes, quien describe en su "Modelo del Estado Completo de Salud" el bienestar como la suma del bienestar emocional, el bienestar psicológico y el bienestar social (Keyes, 2007). Entre 2005 y 2009 Diener amplía sus investigaciones en torno al bienestar subjetivo, incluyendo también componentes de funcionalidad y de contribución con la sociedad (Diener et al., 2010). Por último, Seligman (2012) propone el modelo PERMA constituido por los 5 pilares en los que se sustenta el Florecimiento ("Positive emotions", "Engagement", "Positive Relationships", "Meaning” y "Accomplishments"). En definitiva, la SMP podría definirse como "El concepto que denomina ese algo más que ausencia de enfermedad" (Lluch-Canut, 2008, p. 38); es decir, una entidad que difiere de y significa más que la simple ausencia de enfermedad, es equiparable al bienestar e incluye emociones positivas y negativas, activos y habilidades psicológicos que facilitan la adaptación y habilidades sociales que favorecen el funcionamiento positivo en relación con los otros y con la sociedad.

\section{Salud Mental Positiva como Constructo: Revisión de Teorías}

Para la consecución de este segundo objetivo se reunieron los conceptos relacionados con los aspectos positivos de la salud mental, encontrándose los siguientes: 
felicidad, optimismo, bienestar, bienestar psicológico, bienestar emocional, bienestar social, florecimiento -flourish- y SMP. A continuación, se exploraron los modelos teóricos que sustentan dichos conceptos, con el fin de enumerar las dimensiones que las constituyen (Tabla 1).

Tabla 1 - Conceptos y Dimensiones de los Principales Modelos Teóricos de la Psicología Positiva

\begin{tabular}{|c|c|c|c|}
\hline $\begin{array}{l}\text { Autor y } \\
\text { año }\end{array}$ & Teoría & Concepto & Dimensiones \\
\hline $\begin{array}{l}\text { Jahoda } \\
(1958)\end{array}$ & $\begin{array}{l}\text { Modelo } \\
\text { de Salud } \\
\text { Mental } \\
\text { Positiva }\end{array}$ & $\begin{array}{l}\text { Salud Men- } \\
\text { tal Positiva }\end{array}$ & $\begin{array}{l}\text { 1. Actitudes hacia uno } \\
\text { mismo } \\
\text { 2. Crecimiento y auto- } \\
\text { actualización } \\
\text { 3. Integración } \\
\text { 4. Autonomía } \\
\text { 5. Percepción de la } \\
\text { realidad y sensibilidad } \\
\text { social } \\
\text { 6. Dominio del en- } \\
\text { torno }\end{array}$ \\
\hline $\begin{array}{l}\text { Bradburn } \\
(1969)\end{array}$ & & $\begin{array}{l}\text { Felicidad } \\
\text { Afectos } \\
\text { positivos y } \\
\text { negativos }\end{array}$ & $\begin{array}{l}\text { Escala de balance } \\
\text { afectivo }\end{array}$ \\
\hline $\begin{array}{l}\text { Diener } \\
(1984)\end{array}$ & & $\begin{array}{l}\text { Bienestar } \\
\text { Subjetivo }\end{array}$ & $\begin{array}{l}\text { 1. Balance afectivo: } \\
\text { Presencia de afectos } \\
\text { positivos y ausencia de } \\
\text { afectos negativos } \\
\text { 2. Satisfacción con la } \\
\text { vida }\end{array}$ \\
\hline $\begin{array}{l}\text { Ryff } \\
(1989)\end{array}$ & $\begin{array}{l}\text { Teoría del } \\
\text { Bienestar }\end{array}$ & $\begin{array}{l}\text { Bienestar } \\
\text { Psicológico }\end{array}$ & $\begin{array}{l}\text { 1. Auto-aceptación } \\
\text { 2. Propósito en la vida } \\
\text { 3. Autonomía } \\
\text { 4. Relaciones interper- } \\
\text { sonales positivas } \\
\text { 5. Dominio del en- } \\
\text { torno } \\
\text { 6. Crecimiento per- } \\
\text { sonal }\end{array}$ \\
\hline $\begin{array}{l}\text { Keyes } \\
(1998)\end{array}$ & $\begin{array}{l}\text { Teoría del } \\
\text { Bienestar } \\
\text { Social }\end{array}$ & $\begin{array}{l}\text { Bienestar } \\
\text { Social }\end{array}$ & $\begin{array}{l}\text { 1. Integración social } \\
\text { 2. Aceptación social } \\
\text { 3. Contribución social } \\
\text { 4. Actualización social } \\
\text { 5. Coherencia social }\end{array}$ \\
\hline $\begin{array}{l}\text { Lluch- } \\
\text { Canut } \\
(2002)\end{array}$ & $\begin{array}{l}\text { Modelo } \\
\text { Multifac- } \\
\text { torial de } \\
\text { Salud } \\
\text { Mental } \\
\text { Positiva }\end{array}$ & $\begin{array}{l}\text { Salud men- } \\
\text { tal positiva }\end{array}$ & $\begin{array}{l}\text { 1. Satisfacción personal } \\
\text { 2. Actitud prosocial } \\
\text { 3. Autocontrol } \\
\text { 4. Autonomía } \\
\text { 5. Resolución de } \\
\text { problemas y autoactu- } \\
\text { alización } \\
\text { 6. Habilidades de rel- } \\
\text { ación interpersonal }\end{array}$ \\
\hline $\begin{array}{l}\text { Seligman } \\
(2002)\end{array}$ & $\begin{array}{l}\text { Teoría de } \\
\text { la Felici- } \\
\text { dad }\end{array}$ & $\begin{array}{l}\text { Teoría de la } \\
\text { Auténtica } \\
\text { Felicidad }\end{array}$ & $\begin{array}{l}\text { 1. Emociones positivas } \\
\text { 2. Compromiso } \\
\text { 3. Significado de la } \\
\text { vida }\end{array}$ \\
\hline
\end{tabular}

\begin{tabular}{|c|c|c|c|}
\hline $\begin{array}{l}\text { Keyes } \\
(2007)\end{array}$ & $\begin{array}{l}\text { Teoría del } \\
\text { estado } \\
\text { completo } \\
\text { de salud. } \\
\text { Modelo } \\
\text { de los dos } \\
\text { continu- } \\
\text { um }\end{array}$ & $\begin{array}{l}\text { Floreci- } \\
\text { miento }\end{array}$ & $\begin{array}{l}\text { 1.Afectos positivos } \\
\text { 2. Calidad de vida } \\
\text { percibida } \\
\text { 3. Auto-aceptación } \\
\text { 4. Propósito en la vida } \\
\text { 5. Autonomía } \\
\text { 6. Relaciones interper- } \\
\text { sonales positivas } 7 \text {. } \\
\text { Dominio del entorno } \\
\text { 8. Crecimiento personal } \\
\text { 9. Integración social } \\
\text { 10. Aceptación social } \\
\text { 11. Contribución social } \\
\text { 12. Actualización social } \\
\text { 13. Coherencia social }\end{array}$ \\
\hline $\begin{array}{l}\text { Diener et } \\
\text { al. (2010) }\end{array}$ & & $\begin{array}{l}\text { Floreci- } \\
\text { miento }\end{array}$ & $\begin{array}{l}\text { Desarrolla un concepto } \\
\text { de bienestar que engloba } \\
\text { los } 3 \text { componentes ( } \\
\text { emocional, psicológico y } \\
\text { social) que llama Flore- } \\
\text { cimiento y un instru- } \\
\text { mento de medida }\end{array}$ \\
\hline $\begin{array}{l}\text { Seligman } \\
(2012)\end{array}$ & $\begin{array}{l}\text { Teoría del } \\
\text { Bienestar }\end{array}$ & PERMA & $\begin{array}{l}\text { 1. Emociones Positivas } \\
\text { 2. Compromiso } \\
\text { 3. Relaciones interper- } \\
\text { sonales positivas } \\
\text { 4. Significado de la vida } \\
\text { 5. Establecimiento de } \\
\text { metas }\end{array}$ \\
\hline
\end{tabular}

Por último, se compararon los modelos teóricos y las dimensiones de los constructos con el fin de establecer relaciones categóricas. También se enumeraron los instrumentos desarrollados por dichos autores para la medición de los constructos estudiados.

Atendiendo a los componentes del Bienestar descritos a nivel conceptual (Hedonia y Eudamonia), se clasificaron los modelos teóricos analizados en dos grupos. En el primero (Tabla 2), encontramos aquellos autores que se refieren al concepto de bienestar únicamente de manera parcial.

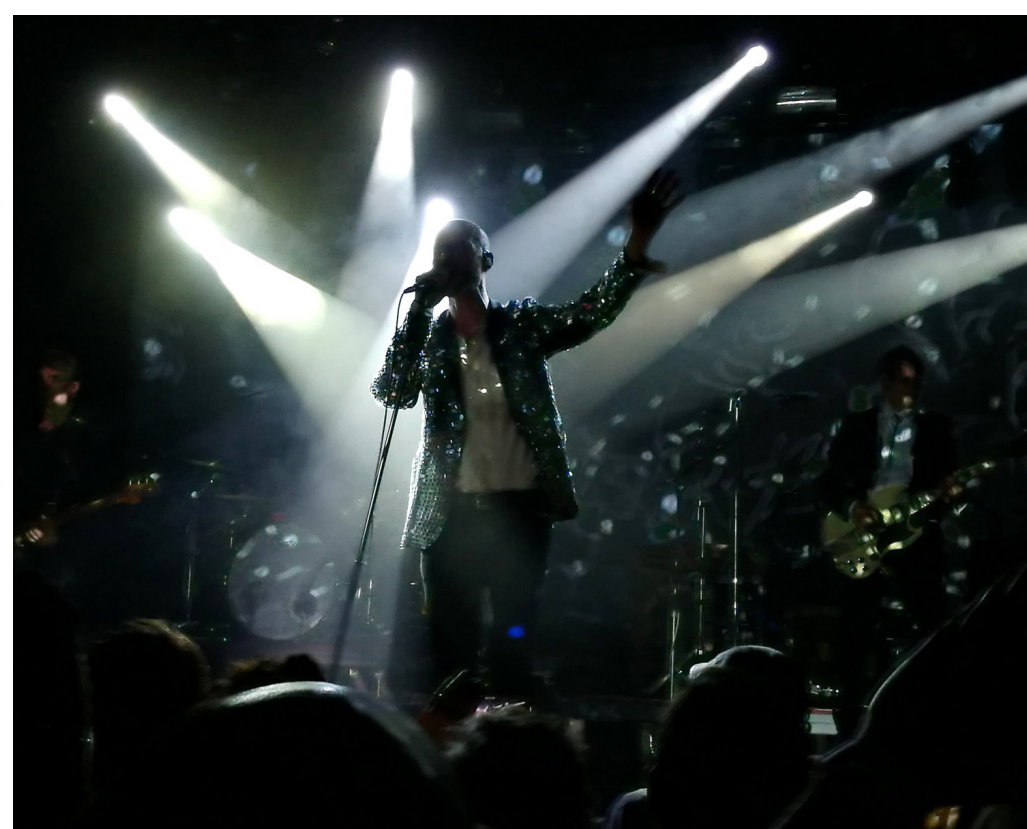


Tabla 2 - Grupo 1-Modelos Parciales de Bienestar

\begin{tabular}{|c|c|c|c|c|c|c|c|c|}
\hline \multicolumn{3}{|c|}{$\begin{array}{l}\text { MODELOS } \\
\text { TEÓRICOS }\end{array}$} & $\begin{array}{l}\text { Jahoda (1958) Modelo } \\
\text { de SMP }\end{array}$ & $\begin{array}{l}\text { Bradburn (1969) } \\
\text { Bienestar Psi- } \\
\text { cológico }\end{array}$ & $\begin{array}{l}\text { Diener (1984) } \\
\text { Teoría del Bien- } \\
\text { estar Psicológico }\end{array}$ & $\begin{array}{l}\text { Ryff (1989) Teoría } \\
\text { del Bienestar Psi- } \\
\text { cológico }\end{array}$ & $\begin{array}{l}\text { Keyes (1998) } \\
\text { Teoría del Bien- } \\
\text { estar Social }\end{array}$ & $\begin{array}{l}\text { Seligman } \\
(2002) \text { Teoría } \\
\text { de la Auténtica } \\
\text { Felicidad }\end{array}$ \\
\hline \multirow{3}{*}{ 至 } & 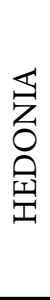 & 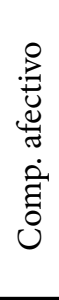 & & $\begin{array}{l}\text { 1. Felicidad } \\
\text { 2. Afectos Posi- } \\
\text { tivos } \\
\text { 3. Afectos Nega- } \\
\text { tivos }\end{array}$ & $\begin{array}{l}\text { 1. Balance afec- } \\
\text { tivo: Presencia de } \\
\text { afectos positivos y } \\
\text { ausencia de afec- } \\
\text { tos negativos } \\
\text { 2.Satisfacción con } \\
\text { la vida }\end{array}$ & & & $\begin{array}{l}\text { 1. Emociones } \\
\text { Positivas } \\
\text { 2. Significado } \\
\text { de la vida }\end{array}$ \\
\hline & \multirow[t]{2}{*}{ 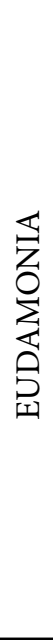 } & 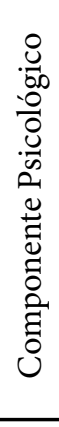 & $\begin{array}{l}\text { 1. Actitudes hacia uno } \\
\text { mismo } \\
\text { 2. Crecimiento y auto- } \\
\text { actualización } \\
\text { 3. Integración } \\
\text { 4.Autonomía } \\
\text { 5. Percepción de la } \\
\text { realidad, empatía y } \\
\text { sensibilidad social } \\
\text { 6. Dominio del entorno }\end{array}$ & & & $\begin{array}{l}\text { 1.Auto-aceptación } \\
\text { 2.Propósito en la } \\
\text { vida } \\
\text { 3.Autonomía } \\
\text { 4.Crecimiento per- } \\
\text { sonal } \\
\text { 5.Relaciones inter- } \\
\text { personales positivas } \\
\text { 6.Dominio del } \\
\text { entorno }\end{array}$ & & 3.Compromiso \\
\hline & & 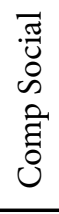 & & & & & $\begin{array}{l}\text { 1. Integración } \\
\text { 2. Aceptación } \\
\text { 3. Contribución } \\
\text { 4. Actualización } \\
\text { 5. Coherencia }\end{array}$ & \\
\hline \multicolumn{3}{|c|}{ ESCALAS } & & $\begin{array}{l}\text { Escala de Bal- } \\
\text { ance Afectivo } \\
\text { (ABS) }\end{array}$ & $\begin{array}{l}\text { Escala de Satisfac- } \\
\text { ción con la vida } \\
\text { (SWLS) }\end{array}$ & $\begin{array}{l}\text { Escala de Bienestar } \\
\text { Psicológico (PWBS) }\end{array}$ & $\begin{array}{l}\text { Escala del } \\
\text { Bienestar Social } \\
\text { (SWBS) }\end{array}$ & \\
\hline
\end{tabular}

En el segundo grupo se incluyeron los modelos teóricos que analizan el concepto de manera global, es decir, que comprenden elementos afectivos, psicológicos y sociales como dimensiones del constructo (Tabla 3).

Tabla 3 - Grupo2-Modelos Globales de Bienestar

\begin{tabular}{|c|c|c|c|c|c|c|}
\hline \multicolumn{3}{|c|}{$\begin{array}{l}\text { MODELOS } \\
\text { TEÓRICOS }\end{array}$} & $\begin{array}{l}\text { Lluch-Canut (2002) } \\
\text { Modelo Multifactorial } \\
\text { de SMP }\end{array}$ & $\begin{array}{l}\text { Keyes (2007) } \\
\text { Teoría del Estado Completo de Salud }\end{array}$ & $\begin{array}{l}\text { Diener (2010) } \\
\text { Florecimiento }\end{array}$ & $\begin{array}{l}\text { Seligman (2012) } \\
\text { Teoría del Bienestar }\end{array}$ \\
\hline \multirow{3}{*}{ 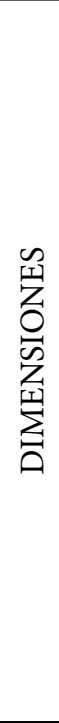 } & $\begin{array}{l}\leftrightarrows \\
\text { Z } \\
\text { 龱 }\end{array}$ & 官: & $\begin{array}{l}\text { 1. Satisfacción Per- } \\
\text { sonal }\end{array}$ & $\begin{array}{l}\text { 1. Afectos positivos } \\
\text { 2. Calidad de vida percibida }\end{array}$ & 1. Significado de la vida & $\begin{array}{l}\text { 1. Emociones Positivas } \\
\text { 2. Satisfacción con la vida }\end{array}$ \\
\hline & \multirow{2}{*}{ 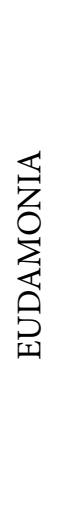 } & 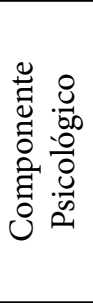 & $\begin{array}{l}\text { 2. Autocontrol } \\
\text { 3. Autonomía } \\
\text { 4. Resolución de } \\
\text { problemas y auto- } \\
\text { actualización } \\
\text { 5. Habilidades de rel- } \\
\text { ación interpersonal }\end{array}$ & $\begin{array}{l}\text { 3. Auto-aceptación } \\
\text { 4. Propósito en la vida } \\
\text { 5. Autonomía } \\
\text { 6. Relaciones interpersonales positivas } \\
\text { 7. Dominio del entorno } \\
\text { 8. Crecimiento personal }\end{array}$ & $\begin{array}{l}\text { 2.Compromiso } \\
\text { 3. Capacidad de resolu- } \\
\text { ción de problemas } \\
\text { 4. Relaciones Interperso- } \\
\text { nales positivas }\end{array}$ & $\begin{array}{l}\text { 3. Compromiso y puesta } \\
\text { en marcha de fortalezas } \\
\text { 4. Logros y establecimiento } \\
\text { de metas }\end{array}$ \\
\hline & & 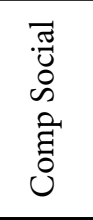 & 6. Actitud Prosocial & $\begin{array}{l}\text { 9. Integración social } \\
\text { 10. Aceptación social } \\
\text { 11.Contribución social } \\
\text { 12.Actualización social } \\
\text { 13. Coherencia social }\end{array}$ & $\begin{array}{l}\text { 5. Contribuir a la Felici- } \\
\text { dad de la comunidad } \\
\text { 6. Ser respetado por la } \\
\text { comunidad }\end{array}$ & $\begin{array}{l}\text { 5.Relaciones interperson- } \\
\text { ales positivas }\end{array}$ \\
\hline \multicolumn{3}{|c|}{ ESCALAS } & $\begin{array}{l}\text { Cuestionario de Salud } \\
\text { Mental Positiva } \\
\text { (CSMP) }\end{array}$ & $\begin{array}{l}\text { Escala de Salud Mental Positiva } \\
\text { (PMH-SF) }\end{array}$ & $\begin{array}{l}\text { Escala de Florecimiento } \\
\text { (FS) }\end{array}$ & PERMA Profile \\
\hline
\end{tabular}


Teniendo en cuenta lo anteriormente descrito, se puede afirmar que la Salud Mental Positiva es un constructo constituido eminentemente por 2 componentes, el hedónico y el eudamónico. El componente hedónico está formado principalmente por la satisfacción con la vida, emociones positivas y negativas y el balance entre ambas. Por otro lado, el componente eudamónico engloba tanto aspectos psicológicos relacionados con la autonomía y resolución de problemas de la vida diaria, como aspectos sociales, que indican el grado de aceptación social y contribución a la sociedad que percibe cada persona, entre otros.

\section{DISCUSIÓN}

De acuerdo a los estudios revisados, se observa una tendencia, iniciada por Lluch-Canut (2002) y consolidada en la segunda década del siglo XXI, a cuestionar los modelos de bienestar existentes hasta el momento y a desarrollar sus propios modelos basados en un concepto globalizador, así como los respectivos instrumentos de medida (Diener et al., 2010; Keyes, 2007; Lluch-Canut, 2002; Seligman, 2012).

En lo referente a la construcción del concepto, todos los autores apoyan la hipótesis de los 2 componentes, Hedonia y Eudamonia, a excepción de Lluch-Canut que no tiene en cuenta dicha distribución al describir su constructo. Si bien es cierto que no existe consenso en los elementos internos que constituirían cada uno de los componentes, el análisis en profundidad de los modelos teóricos ha revelado semejanzas entre los mismos.

Por otro lado, autores como Diener et al. (2010), Huppert y So (2013) o Kobau et al. (2011) proponen la SMP como una alternativa interesante para medir el grado de desarrollo de una nación; Seligman (2008) va más allá al sugerir que podría incluso sustituir al indicador actual, es decir, al Producto Interior Bruto (PIB). Este hecho abre nuevas líneas de investigación que confluyen con las actuales estrategias de los sistemas sanitarios, en los que el nuevo foco de atención se centraría en atender a un número extraordinariamente mayor de clientes potenciales, es decir, la persona sana. Asimismo, es necesario implantar y evaluar programas de intervención para elevar la SMP de la población general y conocer su repercusión frente al desarrollo de enfermedades.

\section{CONCLUSIONES}

La SMP como concepto es un término que, aunque no es reciente, no ha sido tomado en cuenta por la Psicología Positiva hasta hace relativamente pocos años. En esta revisión queda de manifiesto que la propuesta de Lluch-Canut permanece vigente.

Se han identificado numerosos términos que, aunque referidos a ideas similares, no son necesariamente idénticos. Entre otros, se subrayan nociones como las emociones positivas, el optimismo que, aunque pertenecen al paradigma positivo, sólo describen parcialmente la idea de SMP, pudiendo ser consideradas algunas de ellas como componentes de la misma.

Por otro lado, la Salud Mental Positiva como constructo posee un componente hedónico, relacionado con el bienestar emocional, y un componente eudamónico, relacionado con el funcionamiento psicológico de cada persona y su grado de aceptación y contribución social, principalmente. Sólo 2 de los constructos analizados cumplen estas características descritas, que son SMP, recuperado por Lluch-Canut (2002) y Keyes (2007), y el concepto de Florecimiento, planteado por Diener et al. (2010) y Seligman (2012). Todos ellos alternan indistintamente el constructo que estudian con el de Bienestar, aunque sólo Seligman ha utilizado este término en concreto para describir su teoría.

\section{IMPLICACIONES PARA LA PRÁCTICA CLÍNI- CA}

El cambio de paradigma genera nuevas inquietudes en torno a la "etiología de la SMP" de las personas. Por otra parte, enlazando con la idea del ser humano como ser bio-psico-social, comienzan a adquirir mayor importancia los factores circunstanciales y contextuales que juegan un papel en el establecimiento de la SMP. Así, se entiende que la SMP de un individuo no depende exclusivamente de los recursos genéticos, biológicos o psicológicos con los que llega a este mundo, sino que los aspectos sociales constituyen una carga de gran peso en el mismo. Incluso la propia Psicología Positiva supera su propio paradigma individualista para fijarse ahora en un objetivo más amplio, la Salud Mental Positiva y su concepción comunitaria.

Al incidir en el estudio de los aspectos positivos de la Salud Mental, se reconoce la SMP como un campo histórico, socialmente determinado y ne $\neg$ cesario para la promoción de la salud y direccionamiento de las políticas públi $\neg$ cas en salud mental. 


\section{REFERENCIAS BIBLIOGRÁFICAS}

Antonovsky, A. (1996). The salutogenic model as a theory to guide health promotion. Health Promotion International, 11(1), 11-18. Consultado em: https://academic.oup.com/heapro/article-abstract/11/1/11/582748

Botello, B., Palacio, S., García, M., Margolles, M., Fernández, F., Hernán, M., ... Cofiño, R. (2013). Metodología para el mapeo de activos de salud en una comunidad. Gaceta Sanitaria, 27(2), 180-183. Doi: org/10.1016/j.gaceta.2012.05.006

Bradburn, N. M. (1969). The structure of psychological well-being. Oxford: Aldine.

Diener, E. (1984). Subjective Well-Being. Psychological Bulletin, 95(3), 542-575. Doi: org/10.1007/978-90481-2350-6_2

Diener, E., Wirtz, D., Tov, W., Kim-Prieto, C., Choi, D. W., Oishi, S., ... Biswas-Diener, R. (2010). New wellbeing measures: Short scales to assess flourishing and positive and negative feelings. Social Indicators Research, 97(2), 143-156. Doi: org/10.1007/s11205-0099493-y

Huppert, F. y So, T. (2013). Flourishing across Europe : Application of a new conceptual framework for defining well-being. Social Indicators Research, 110(3), 837-861. Doi: 10.1007/s11205-011-9966-7

Jahoda, M. (1958). Current concepts of positive mental health. New York: Basic Book.

Keyes, C. (1998). Social well-being. Social Psychology Quarterly, 61(2), 121-140. Doi: 10.2307/2787065

Keyes, C. (2007). Promoting and protecting mental health as flourishing: A complementary strategy for improving national mental health. American Psychologist, 62(2), 95-108. Doi: 10.1037/0003-066X.62.2.95

Keyes, C. y Simoes, E. (2012). To flourish or not: Positive mental health and all-cause mortality. American Journal of Public Health, 102(11), 2164-2172. Doi: 10.2105/AJPH.2012.300918
Kobau, R., Seligman, M., Peterson, C., Diener, E., Zack, M., Chapman, D., ... Thompson, W. (2011). Mental health promotion in public health: Perspectives and strategies from positive psychology. American Journal of Public Health, 101(8), 1-9. Doi: 10.2105/ AJPH.2010.300083

Lluch-Canut, M. (2002). Evaluación emprírica de un modelo de Salud Mental Positiva. Salud Mental, 25(4), 42-55. Doi: 10.4090/juee.2008.v2n2.033040

Lluch-Canut, M. (2008). Concepto de Salud Mental Positiva: factores relacionados. En J. Fornés y J. Gómez (Eds.), Recursos y programas para la Salud Mental. Enfermería PSicosocial II (37-68). Barcelona: Enfo Ediciones para FUDEN.

Organización Mundial de la Salud. (2013). Plan de acción sobre Salud Mental 2013-2020.

Organización Mundial de la Salud. (2017). Trastornos mentales. Nota descriptiva.

Orem, D. y Vardiman, E. (1994). Orem's nursing theory and positive mental health: Practical considerations. Nursing Science Quarterly, 8(4), 165-173. Doi: org/0803973233

Ryff, C. (1989). Happiness is everything, or is It - explorations on the meaning of psychological well-being. Journal of Personality and Social Psychology, 57(6), 1069-1081. Doi: org/10.1037/0022-3514.57.6.1069|

Seligman, M. (2002). Authentic happiness: Using the new positive psychology to realize your potential for lasting fulfillment. Nueva york: Simon y Schuster Seligman, M. (2008). Positive health. Applied Psychology, 57(SUPPL. 1), 3-18. Doi: org/10.1111/j.14640597.2008.00351.x

Seligman, M. (2012). Flourish: A visionary new understanding of happiness and well-being. Nueva york: Simon y Schuster

Wand, T. (2013). Positioning mental health nursing practice within a positive health paradigm. International Journal of Mental Health Nursing, 22(2), 116124. Doi: org/10.1111/j.1447-0349.2012.00848.x 